\title{
Instrumentation Optimization for Positron Emission Mammography
}

\author{
William W. Moses ${ }^{\mathrm{a},{ }^{*}}$ and Jinyi $\mathrm{Qi}^{\mathrm{a}}$ \\ ${ }^{a}$ Lawrence Berkeley National Laboratory, 1 Cyclotron Rd., Berkeley, CA 94720, USA
}

Elsevier use only: Received date here; revised date here; accepted date here

\begin{abstract}
The past several years have seen designs for PET cameras optimized to image the breast, commonly known as Positron Emission Mammography or PEM cameras. The guiding principal behind PEM instrumentation is that a camera whose field of view is restricted to a single breast has higher performance and lower cost than a conventional PET camera. The most common geometry is a pair of parallel planes of detector modules, although geometries that encircle the breast have also been proposed. The ability of the detector modules to measure the depth of interaction (DOI) is also a relevant feature. This paper finds that while both the additional solid angle coverage afforded by encircling the breast and the decreased blurring afforded by the DOI measurement improve performance, the ability to measure DOI is more important than the ability to encircle the breast.
\end{abstract}

Keywords: Positron Emission Mammography; Breast Cancer; PEM Camera Design and Optimization

\section{Introduction}

An increasing number of PET cameras optimized to image the breast have been proposed or constructed [1-9]. These cameras, commonly known as Positron Emission Mammography or PEM cameras, restrict the field of view to a single breast, and are expected to have higher performance and lower cost than a conventional PET camera. By placing the detectors close to the breast, the PEM geometry is able to subtend more solid angle around the breast than a conventional PET camera. In addition, gamma rays emitted in the breast have to pass through at most one attenuation length $(\sim 10 \mathrm{~cm})$ of tissue in the PEM geometry, but may have to travel through as much as four attenuation lengths of tissue in a conventional PET camera. These two factors significantly increase the sensitivity (the detected coincident event rate per unit activity in the field of view) in the PEM geometry.

There are two additional design features that have a large affect on the performance of PEM cameras the geometric efficiency for detecting activity that is within the field of view and whether the detector module is capable of measuring the depth of interaction (DOI, described later). While having a high efficiency and the ability to measure DOI are both desirable, they come at some cost, and so we must estimate their benefits in order to maximize the cost / performance tradeoff. Therefore, this paper

\footnotetext{
" Corresponding author. Tel.: +1-510-486-4432; fax: +1-510-486-4768; e-mail: wwmoses@lbl.gov.
} 


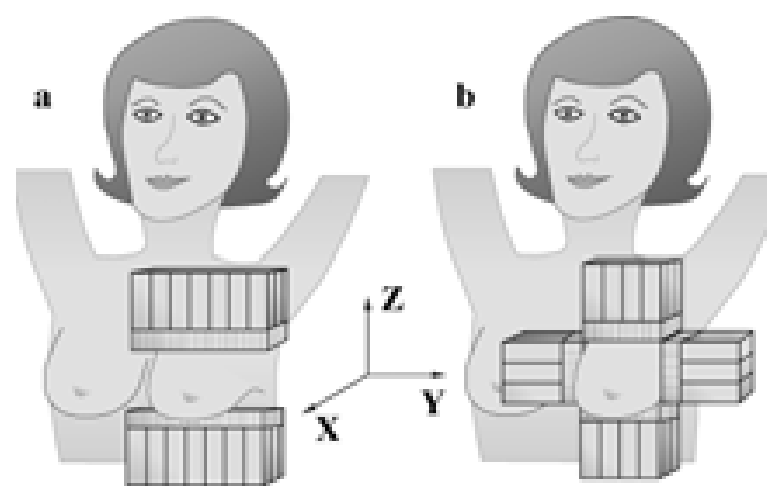

Figure 1. The two PEM camera geometries simulated in this paper. The parallel plane geometry is shown in a), the rectangular geometry in b).

examines several PEM camera designs in order to identify which design tradeoffs have the greatest affect on the imaging performance.

\section{Camera Design}

Four hypothetical camera designs are examined to identify the most important features. We examine the four combinations implied by two geometries (with different geometrical acceptance) and two DOI measurement ability options (with and without).

\subsection{Efficiency}

The efficiency is dominated by the geometric acceptance, which is defined as the fraction of the $4 \pi$ solid angle surrounding a source (placed at an arbitrary location within the camera) in which both back-to-back emanations from the source impinge on the detector. This geometric acceptance does not include the affect of attenuation of the gamma rays before they reach the detectors or detector efficiency, although clearly the detector efficiency must be high in order to obtain high overall detection efficiency.

We start with a "standard" PEM camera with the geometry shown in Figure 1a. It consists of two parallel planes of detectors, each plane being $17.5 \mathrm{~cm}$ wide and $7.5 \mathrm{~cm}$ deep, with a spacing of $7.5 \mathrm{~cm}$ between planes. We call this the P-PEM (for parallel plane) geometry. The other geometry, known as RPEM (for rectangular) consists of two pairs of parallel planes arranged in a rectangular geometry, as shown in Figure 1b. The patient port is $10.0 \mathrm{~cm}$ wide, $7.5 \mathrm{~cm}$ wide, and $7.5 \mathrm{~cm}$ deep. Note that the R-PEM design can be constructed with the same volume of detector material as the P-PEM design, but subtends a greater solid angle than the P-PEM camera..

For all cameras, the detectors are assumed to have $3 \mathrm{~mm}$ spatial resolution, to be $30 \mathrm{~mm}$ deep, and to be made of LSO scintillator material [10] which has an attenuation length for $511 \mathrm{keV}$ photons of $1.2 \mathrm{~cm}$. All valid time coincidences between any detector element in one plane and any detector element in any of the other planes are kept. The orientation of the coordinate axes is also shown in Figure 1, with the origin located at the center of the field of view.

\subsection{Depth of Interaction}

In both the P-PEM and R-PEM geometries, an inherent degradation exists because the object to be imaged is close to the detector modules. As Figure 2 shows, many gamma rays penetrate a significant distance into the detectors before they interact and are detected. If the interaction depth within a detector element is not measured (as with conventional PET detector modules), then the interaction position is assigned to the front face of the detector element that the interaction occurs in. A line joining the two such assigned points may not pass through the actual source position, resulting in mis-positioning errors and degradation of the spatial resolution.

This distortion can be reduced by reducing the thickness of the detectors. However, this decreases the fraction of emitted gamma rays that interact in the detectors, and so reduces the single gamma ray detection efficiency. As two gamma ray detections are required for a PEM event, the overall detection efficiency is the square of the single gamma ray detection efficiency, so a camera with 1 interaction length of detector will have an efficiency that is less than half that of a camera with 3 attenuation lengths of detector. If the depth of interaction (DOI) is measured (leaving the detector 3 attenuation lengths thick), the line joining the two measured interaction positions will pass through the actual source position, and the high detection efficiency will be maintained with no mis-positioning errors. 


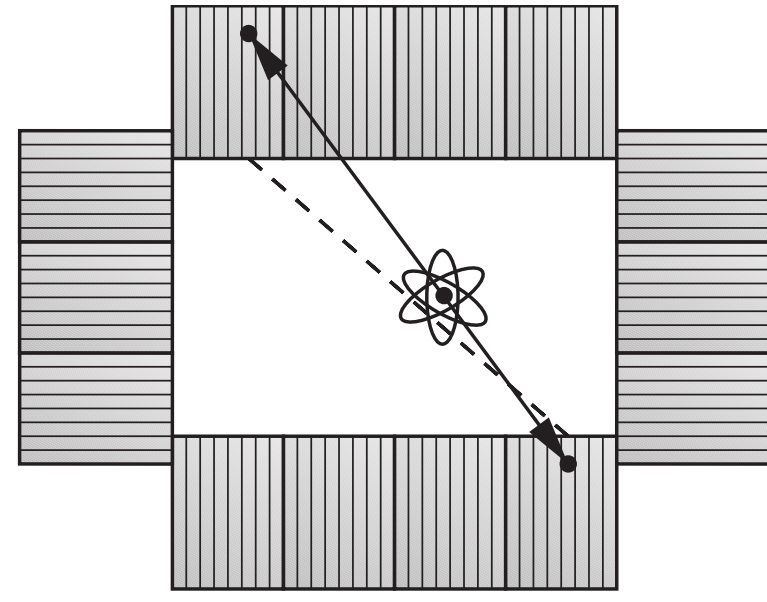

Figure 2. If the interaction position of gamma rays that penetrate into the detector module is assigned to the front face of the detector element, mis-positioning errors occur as the line connecting these points does not go through the source (the dotted line). If the interaction depth in the detector is measured, then the position is no longer assigned to the front face and the mis-positioning error is eliminated (solid line).

We therefore consider cameras with and without DOI measurement capability. For the non-DOI case, we assume that all interactions are assigned to the front face of the detector element that the interaction took place in. For the DOI case, we assume that the $30 \mathrm{~mm}$ depth of the scintillator crystal is divided into eight sections in depth (each $3.75 \mathrm{~mm}$ deep), and that the detector is able to properly identify the section that an interaction occurs in.

\section{Performance Comparison}

\subsection{The Fisher Information Matrix}

Comparing PEM camera designs poses several challenges. First, a task and a performance measure for this task must be defined. For example, we might compute the signal to noise ratio for a spherical "tumor" in a uniform background. Given the heterogeneity of tumors in patients, a single task is usually woefully inadequate to characterize performance. In the spherical tumor example, we should measure a variety of "tumors" with different diameters, signal to background ratios, positions within the camera, and overall activity levels.

A straightforward method to do this is Monte Carlo simulation. However, such simulations are computationally intensive $\left(10^{10}-10^{11}\right.$ positron annihilations per image must be generated), greatly restricting how many different activity distributions can be simulated. In addition, the results depend on the reconstruction algorithm that is used, which is often problematic, as optimized reconstruction algorithms usually haven't been developed when one is evaluating novel geometries.

We therefore perform these comparisons using the Fisher information matrix, which is computed analytically using the (geometrically determined) forward projection matrix from image space to detector space. Once this matrix has been computed for a camera design, results for an arbitrary source distribution can be obtained merely by multiplying the Fisher information matrix by a matrix representing the source distribution, which is much faster than running Monte Carlo simulations.

While the method is described in more detail in [11], the Fisher information matrix characterizes how easily a change of one parameter in the source distribution can be identified from the measured data. To be easily identified, the change at one voxel must make significant contribution to the measurements (as compared with the background noise), and such contribution must not be (strongly) correlated with contributions from other voxels. The Fisher information matrix presents these quantities in a matrix form: the diagonal elements measure the significance of the contribution from each voxel, and the off-diagonal elements measure the correlations. Thus, it can assess the limit of attainable image quality without performing a reconstruction. It can also be used to compute figures of merit for task performance. Here we will use the Fisher information matrix to determine the signal to noise ratio and to compute the lesion detectability for spherical tumors of various diameters and contrast.

\subsection{Appearance of the Fisher Information Matrix}




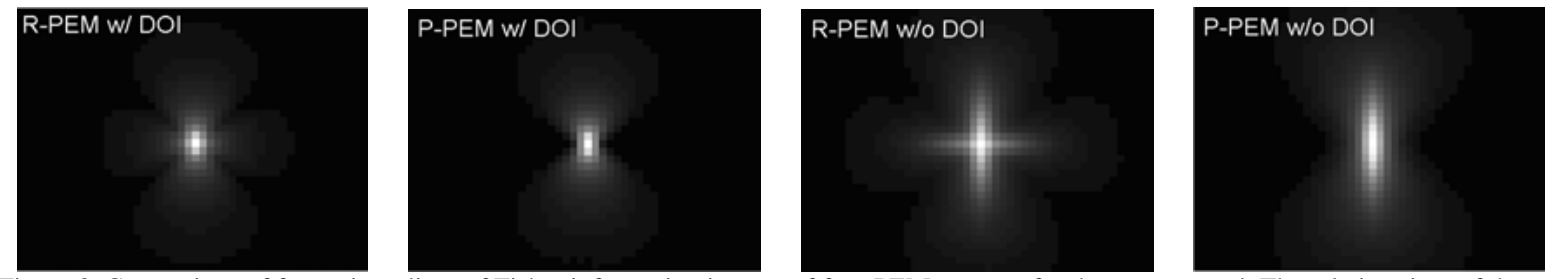

Figure 3. Comparison of front view slices of Fisher information images of four PEM systems for the center voxel. The relative sizes of the maximum values are, from left to right, 1.00 (R-PEM w/ DOI), 0.86 (P-PEM w/ DOI), 0.50 (R-PEM w/o DOI), and 0.37 (P-PEM w/o DOI).

Figure 3 shows a portion of the Fisher information matrix $\boldsymbol{F}$ for the four systems considered. The details for the $\boldsymbol{F}$ computation are given in [11]. The source is assumed to be located at the central voxel in each camera, and the view shown is of a vertical $(x=0)$ slice through the center of the $3 \mathrm{D}$ volume (i.e. a view from the front).

The images in Figure 3 show the correlation between the voxel containing a source (in this case the center voxel) and the rest of the voxels. An ideal system would have a very large value for the voxel containing the source - this indicates that the system is sensitive to small changes in this voxel. This value is also proportional to the $\mathrm{SNR}^{2}$ of detecting a small lesion in this voxel. An ideal system would also have zero values for all voxels that do not contain the source, indicating that there are no correlations and the system can easily distinguish a source placed in one voxel from a source placed in another voxel. In practice, correlations do exist and the off-diagonal elements $\left(F_{j k} ; j \neq k\right)$ are non-zero. Their effect depends on the specific application of the system. For some tasks these non-zero values can even be helpful (see [12] for more discussion).

The R-PEM with DOI has the largest $F_{j j}$., although the $F_{j j}$ of the P-PEM with DOI is $86 \%$ of that of the rectangular R-PEM. For the systems without DOI, the $F_{j j}$ of the R-PEM drops to $50 \%$, and the P-PEM drops to $37 \%$. The Fisher information images for the non-DOI systems are elongated in the vertical and horizontal (R-PEM only) directions because of penetration into the crystals - this penetration (effectively placing activity in voxels other than the one that it originated in) also reduces the diagonal components $F_{j j}$. As mentioned earlier, such elongation can be reduced by using shorter detector crystals, but this would reduce the overall sensitivity.

\subsection{Signal to Noise Ratio}

The Fisher information matrix can be used to compute the signal to noise ratio, as described in [11]. Figure 4 compares of the SNRs for lesion detection as functions of the lesion size for all four systems. The simulated lesions are spherical and are located at the center of the FOV. The contrast of the lesion ([lesion activity - background] / background) is one. Figure 4a shows that all SNRs increase as the lesion size increases. The relative performance of the four systems, normalized by the SNR of the R-PEM with DOI, is shown in Figure 4b. The SNR of the PPEM with DOI is about $90 \%$ of the R-PEM with DOI. For lesions less than $9 \mathrm{~mm}$ diameter, the order of the SNRs of the four systems is R-PEM with DOI $>$ P-PEM with DOI > R-PEM w/o DOI > P-PEM w/o DOI. For lesions with diameter larger than $9 \mathrm{~mm}$, the SNR of the R-PEM w/o DOI is greater than the SNR of the P-PEM with DOI. The SNR is proportional to the lesion contrast, so when the contrast changes, the curves in Figure 4a move up or down accordingly, but the curves in Figure $4 \mathrm{~b}$ stay the same.

These results indicate that for lesions $<9 \mathrm{~mm}$ diameter, DOI measurement capability is more important than the additional geometric sensitivity that R-PEM affords, but for lesions $>9 \mathrm{~mm}$ diameter, the higher efficiency is more valuable. This can be understood as follows. The DOI capability mainly enhances the spatial resolution, while the R-PEM geometry mainly enhances the detection efficiency. With small tumors the enhanced spatial resolution is most important, as the blurring caused by penetration spreads the activity into many adjacent voxels, making it difficult to see above background. The magnitude of this blurring is approximately $1 \mathrm{~cm}$ (the attenuation length of LSO), so when the tumor gets 

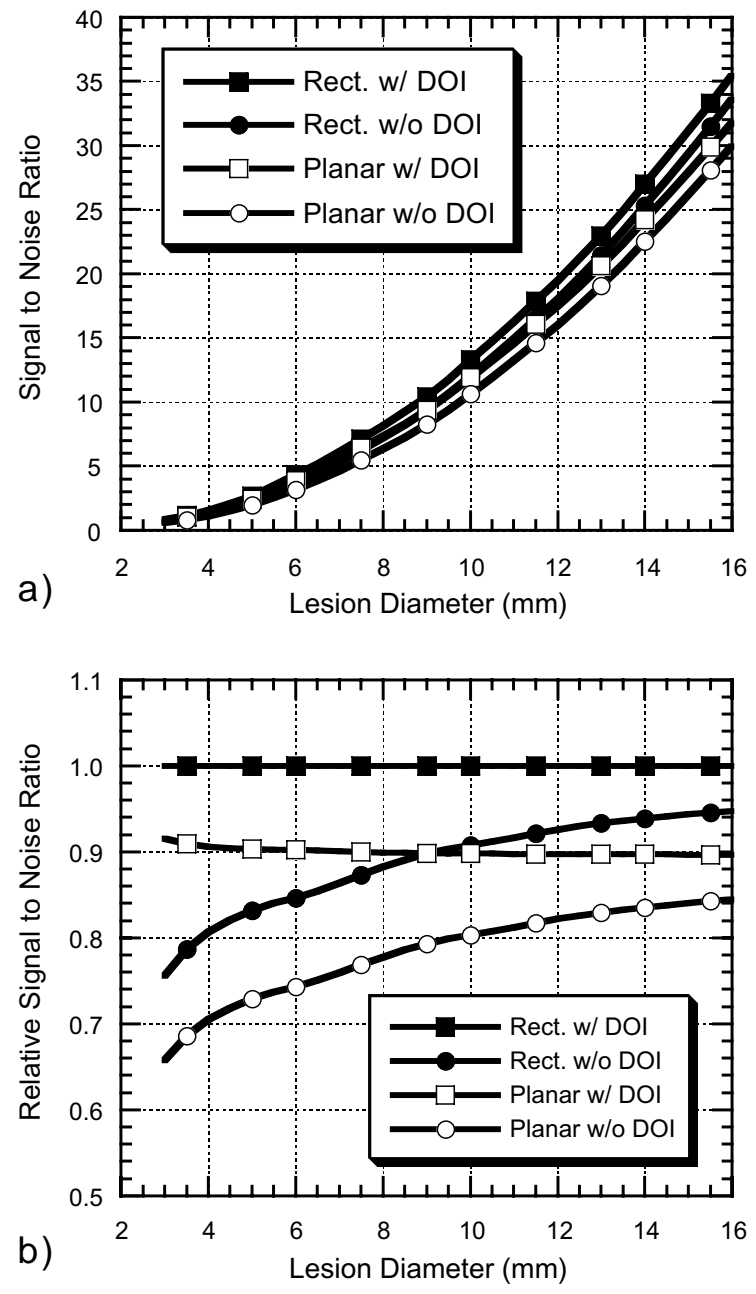

Figure 4. (a) Plots of SNR vs. lesion size for all four systems. (b) The relative SNR of the other three systems when normalized by the SNR of the R-PEM with DOI.

greater than this size, the effects of the blurring are reduced and the increased efficiency becomes more valuable.

\subsection{Tumor Detectability}

While the signal to noise ratio is a useful measure, it does not adequately describe the clinical imaging setting - a more relevant measure is lesion detectability. To illustrate this point, for a $15 \mathrm{~mm}$ diameter tumor Figure 4 shows a higher signal to noise ratio for the R-PEM w/ DOI than any of the other camera designs, suggesting that it may be the best choice. However, the SNR is $>25$ for all of these camera geometries, and so it could be argued that they all have similar performance, as they all would be able to detect the tumor easily.

To estimate tumor detectability, we use the area under the curve (AUC) of the receiver operator characteristic (ROC) curve. The ROC curve quantifies how frequently an observer correctly identifies the tumor. While this curve is traditionally obtained using live humans looking at images, computer models that predict human behavior have been developed. With data similar to that in Figure 4, these computer models have been shown to correlate well with human performance, and so we use that data to compute the AUC (this is described in more detail in [11]). Figure 5 shows the plots of the AUC as a function of the lesion size for all four systems. In Figure 5a, the true contrast of the lesion is $1 / 4$ and in Figure 5b, the contrast is one. As expected, the AUC increases as the size of lesion increases. In addition, for the lesion with contrast of 1/4 (Figure 5a), significant differences among the AUCs of the four systems can be seen when lesion size is between $6 \mathrm{~mm}$ and $9 \mathrm{~mm}$, whereas for the lesion with contrast of one (Figure 5b), such differences can only be seen when lesion size is between $3 \mathrm{~mm}$ and $5 \mathrm{~mm}$.

These results do not imply that PEM cameras will easily detect 3-5 mm diameter tumors in humans. The task modeled is whether a uniform, spherical tumor is visible (above a uniform background) at a known location. Clinical diagnosis is more difficult as the tumor is irregularly shaped, the background is nonuniform, and the position of the tumor is usually not known. However, we expect that the general trend of these results (that small tumors are better observed with P-PEM with DOI than with R-PEM w/o DOI) will hold true in clinical imaging.

\section{Non-Instrumentation Issues}

Some of the critical limitations to PEM have nothing to do with camera design. For example, there is considerable interest in detecting small $(3 \mathrm{~mm}$ diameter and below) tumors. However, small tumors will contain extremely low absolute amounts of activity, and so may be very difficult to observe above the background activity level. Assuming a $10 \mathrm{mCi}$ injection into a $75 \mathrm{~kg}$ patient and a $3: 1$ tumor 

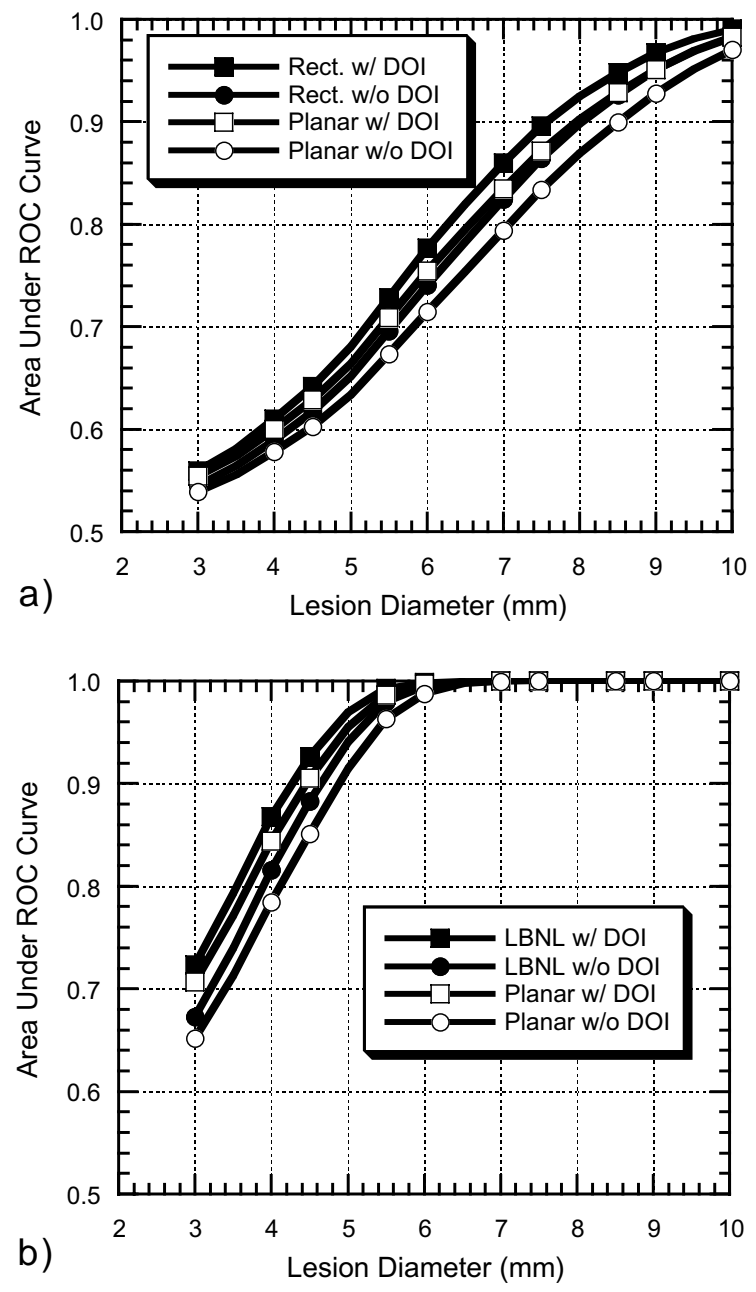

Figure 5. Plots of AUC vs. lesion size for all four systems. (a) Lesion with true contrast of $1 / 4$; (b) lesion with true contrast of 1 .

to normal tissue uptake ratio (a typical value for fluoro-deoxyglucose, which is the most commonly used radiotracer for breast cancer), the expected activity concentration is $150 \mathrm{nCi} / \mathrm{cc}$ in normal tissue and $500 \mathrm{nCi} / \mathrm{cc}$ in tumors. This implies that during a 10 minute acquisition time there would be only 13,000 annihilations in a $3 \mathrm{~mm}$ diameter tumor, as compared to 500,000 annihilations in a $1 \mathrm{~cm}$ diameter tumor and 160 million annihilations in the remainder of the $7.5 \mathrm{~cm} \times 7.5 \mathrm{~cm} \times 10 \mathrm{~cm}$ field of view. Thus, imaging small tumors will be difficult because the volume (and hence number of annihilations) scales as the cube of the tumor diameter.

In addition, there is significant patient to patient variation in the tumor activity concentration (or tumor to normal tissue ratio). The cause for this is not understood - a recent study searched for correlations between the tumor SUV (standard uptake value, which is effectively a measure of the tumor to normal tissue ratio) for fluoro-deoxyglucose and over a dozen different histological and pathological measures of tumor characteristics (e.g., size, grade, vascularity, estrogen and progesterone receptor status, mitotic figure, etc.) and either weak or no correlation was observed with each measure [13]. Thus, it is possible that an impeccably designed PEM camera will be unable to image a breast cancer tumor merely because the tumor, for unknown reasons, has a low radiotracer uptake.

Finally, the exact role of PEM in clinical diagnosis and treatment is uncertain. It is likely to be too expensive to replace $\mathrm{x}$-ray mammography for routine screening, and while its diagnostic accuracy is similar to that of biopsy (PET has $<10 \%$ false negative and false positive fractions for $>1 \mathrm{~cm}$ diameter tumors, while biopsy has a false negative fraction of $\sim 10 \%$ and a false positive fraction of $0 \%$ ), a new technology usually needs to have superior (not merely comparable) performance to replace an existing technology. PEM's limited field of view also limits its utility for staging (determining how far the cancer has spread) or treatment follow-up. While the exact role is yet undetermined, we feel that there are likely to be valuable clinical uses for PEM, such as routine screening for the $\sim 10 \%$ of women for which $x$-ray mammography is unsuitable (e.g., those with mammographically dense breasts, implants, or scarring due to previous biopsy or surgery) or as a non-invasive adjunct to biopsy. We further believe that it is important to develop PEM cameras and perform clinical trials with them. As with any new technology, its optimal roles are not likely to be recognized until they have been used.

\section{Conclusions}

PEM offers significantly higher sensitivity for radiation sources in the breast than conventional PET cameras, mainly because of significantly increased solid angle coverage and reduced attenuation in the patient. There are several design features, notably increased solid angle coverage due to encircling the 
breast and incorporating detector modules that measure the depth of interaction, that can be implemented in PEM cameras that could improve its performance. Using the Fisher information matrix, we have explored the performance difference for four different combinations of these design features. By estimating the signal to noise ratio and tumor detectability for spherical lesions of various sizes and contrasts, we determined that, as expected, the rectangular (R-PEM) geometry with DOI capability had the best performance and the parallel plane (PPEM) geometry w/o DOI had the worst. As the PPEM geometry with DOI outperformed the R-PEM geometry w/o DOI, we conclude that DOI capability is more important than the increased geometric efficiency afforded by the rectangular geometry, especially for small tumors. Finally, there are significant limitations due to non-instrumental effects, such as the absolute amount of radiotracer that is absorbed by the tumor and the uncertain niche for PEM in clinical diagnosis and treatment.

\section{Acknowledgments}

We would like to thank Dr. Ronald Huesman, Dr. Stephen Derenzo, Dr. Thomas Budinger, and Dr. Jennifer Huber of Lawrence Berkeley National Laboratory for many useful discussions. This work was supported in part by the Director, Office of Science, Office of Biological and Environmental Research, Medical Science Division of the U.S. Department of Energy under Contract No. DE-AC0376SF00098, in part by the National Institutes of Health, National Cancer Institute under grant No. R01-CA67911, and in part by National Institutes of Health, National Heart, Lung, and Blood Institute under grant No. P01-HL25840. Reference to a company or product name does not imply approval or recommendation by the University of California or the U.S. Department of Energy to the exclusion of others that may be suitable.

\section{References}

[1] C. J. Thompson, K. Murthy, R. L. Clancy and others, "Imaging performance of a PEM-I: A high resolution system for positron emission mammography," IEEE Nucl Sci Symp and Med Imag Conf Rec, vol. 2, pp. 1074-1078, 1995.

[2] W. W. Moses, T. F. Budinger, R. H. Huesman and S. E. Derenzo, "PET camera designs for imaging breast cancer and axillary node involvement," J. Nucl. Med., vol. 36, pp. 69P, 1995.

[3] I. Weinberg, S. Majewski, A. Weisenberger, A. Markowitz, L. Aloj, et al., "Preliminary results for positron emission mammography - real-time functional breast imaging in a conventional mammography gantry," Euro. J. Nucl. Med., vol. 23, pp. 804-806, 1996.

[4] R. Freifelder and J. S. Karp, "Dedicated PET scanners for breast imaging," Physics in Medicine and Biology, vol. 42, pp. 2463-2480, 1997.

[5] M. B. Williams, R. M. Sealock, S. Majewski and A. G. Weisenberger, "PET detector using waveshifting optical fibers and microchannel plate PMT with delay line readout," IEEE Trans. Nucl. Sci., vol. 45, pp. 195-205, 1998.

[6] W. Worstell, O. Johnson, H. Kudrolli and V. Zavarzin, "First results with high-resolution PET detector modules using wavelength-shifting fibers," IEEE Trans Nucl Sci, vol. 45, pp. 2993-2999, 1998.

[7] N. K. Doshi, Y. P. Shao, R. W. Silverman and S. R. Cherry, "Design and evaluation of an LSO PET detector for breast cancer imaging," Medical Physics, vol. 27, pp. 1535-1543, 2000.

[8] K. Murthy, M. Aznar, A. M. Bergman, C. J. Thompson, J. L. Robar, et al., "Positron emission mammographic instrument: Initial results," Radiology, vol. 215, pp. 280-285, 2000.

[9] R. R. Raylman, S. Majewski, R. Wojcik, A. G. Weisenberger, B. Kross, et al., "The potential role of positron emission mammography for detection of breast cancer. A phantom study," Medical Physics, vol. 27, pp. 1943-1954, 2000.

[10] C. L. Melcher and J. S. Schweitzer, "Cerium-doped lutetium orthosilicate: a fast, efficient new scintillator," IEEE Trans. Nucl. Sci., vol. NS-39, pp. 502-505, 1992.

[11] J. Qi, C. Kuo, R. H. Huesman, G. J. Klein, W. W. Moses, et al., "Comparison of rectangular and dual-planar positron emission mammography systems," IEEE Trans. Nucl. Sci., vol. NS-49, pp. 2089-2096, 2002.

[12] H. H. Barrett, J. L. Denny, R. F. Wagner and K. J. Myers, "Objective assessment of image quality. II. Fisher information, Fourier crosstalk, and figures of merit for task performance," J. Opt. Soc. Amer., vol. A-12, pp. 834-852, 1995.

[13] N. Avril, M. Menzel, J. Dose, M. Schelling, W. Weber, et al., "Glucose metabolism of breast cancer assessed by F-18-FDG PET: Histologic and immunohistochemical tissue analysis," $J$. Nucl. Med., vol. 42, pp. 9-16, 2001. 